hep-ph/0201119

\title{
SPIN AND OTHER ASPECTS OF GENERALIZED PARTON DISTRIBUTIONS巴
}

\author{
M. Diehl $\left.\right|^{2}$ \\ Institut für Theoretische Physik E, RWTH Aachen, 52056 Aachen, Germany
}

\begin{abstract}
I discuss how generalized parton distribution probe various aspects of QCD bound states. Topics include the interplay between transverse and longitudinal structure, quantum mechanical interference, orbital angular momentum, helicity flip, and higher-spin targets.
\end{abstract}

\section{Before talking about spin}

My assignment is to speak about spin aspects of generalized parton distributions. Before doing so I would however like to point out some of the information they contain even if the spin degrees of freedom are all averaged over.

The usual parton densities we are accustomed with are expectation values of quark or gluon operators for a hadron state, and generalized parton distributions [1, 2, 3, 4] are their natural extension to non-forward kinematics. This is readily seen from the diagrams for Compton scattering in the deeply virtual limit (Fig. 1). Since an off-shell photon turns into an on-shell one, the two proton states in the hadronic matrix element must have different momenta. A useful set of variables describing the kinematics are $x$ and $\xi$, parameterizing longitudinal momentum fractions in a frame where both protons move fast, and the Mandelstam invariant $t=\left(p^{\prime}-p\right)^{2}$, which one can trade for the transverse momentum transfer $\left(p^{\prime}-p\right)_{\perp}$. This tells us immediately that we are in a position to probe the transverse and the longitudinal structure of a fast moving proton in a correlated manner.

Setting $\xi$ and $t$ to zero one recovers the usual parton distributions, which have the intuitive interpretation of a density in longitudinal parton momentum. Burkardt [5] has shown that for $\xi=0$ we retain a density interpretation if we perform a Fourier transformation from $\left(p^{\prime}-p\right)_{\perp}$ to impact parameter, which measures the transverse distance of the struck quark from the proton's center. Generalized parton distributions at $\xi=0$ thus describe the transverse distribution of partons with a given longitudinal momentum fraction $x$. Going to

\footnotetext{
${ }^{1}$ Talk given at Pacific Spin 2001, Beijing, China, 8-13 Oct. 2001. To appear in the Proceedings (Int. J. Mod. Phys. A)

2Email: mdiehl@physik.rwth-aachen.de
} 
nonzero $\xi$ we no longer have this density interpretation. I see this as a blessing rather than a curse: after all QCD is a quantum theory, and it may well be that to understand its bound states we will have to go beyond classical probabilities. In any case, generalized parton distributions probe the transverse and longitudinal structure of the proton in a way that has recently been described in the language of photography [6].

(a)

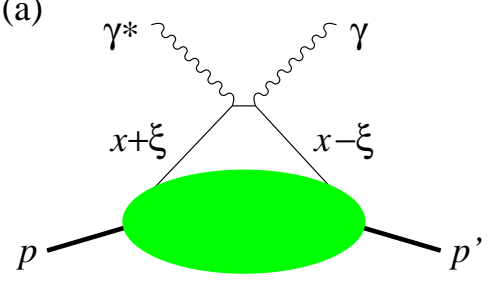

(b)

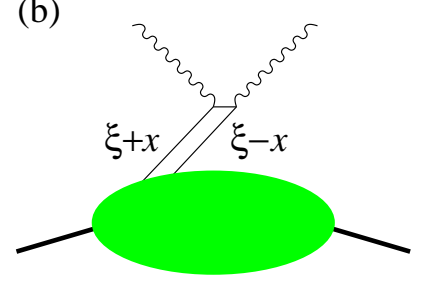

Figure 1: One of the leading diagrams for virtual Compton scattering in the limit of infinite photon virtuality $Q^{2}$ at fixed Bjorken variable $x_{B}$ and invariant momentum transfer $t$. A second diagram is obtained by interchanging the photon vertices. The shaded blob represents a generalized quark distribution. Cases (a) and (b) correspond to different regions of the loop integration over $x$.

The meaning of generalized parton distributions at nonzero $\xi$ can be elucidated in terms of light-cone wave functions [7]. Barring possible subtleties of the light-cone gauge [8], one can write the usual parton densities as squared wave functions, summed over all configurations containing a parton with given momentum fraction $x$. Going to $\xi \neq 0$ we get the product of wave functions corresponding to different momentum fractions of the specified parton. We thus go from classical probabilities to quantum mechanical correlations, or interference terms. Nonzero $\xi$ further opens up a kinematical regime where the distributions describe not the emission and reabsorption of one parton (Fig. 17a) but rather the emission of two partons (Fig. 1 $\mathrm{b}$ ). One then probes $q \bar{q}$ or gluon pairs in the target wave function. Sum rules tell us that the two regimes are intimately connected: both contribute to the integral of the distributions over $x$ at given $\xi$ and $t$, which gives the elastic proton form factors.

These sum rules remind us that quantities like the Dirac form factor $F_{1}(t)$ also contain information about the proton size. From the above discussion it follows that they give the transverse distribution of quarks, now averaged over all longitudinal momenta. This is the opposite reduction of information than the one we encountered in the usual parton densities. Generalized densities combine and interrelate these two partial descriptions. Note also that there are not many pointlike probes providing local currents for a direct measurement of form factors in the nucleon. In particular there is none for gluons to tell us about their transverse location compared to quarks. Such information may be gained in suitable diffractive processes where generalized gluon distributions are enhanced over the quark ones. 
We see that, even before talking about spin, we have access to a much more detailed image of a hadron than the essentially one-dimensional projection of usual parton densities and the information from form factors.

\section{The two roles of spin}

Let us now investigate the spin degrees of freedom in deeply virtual Compton scattering and in exclusive meson production $\gamma^{*} p \rightarrow M p$, or in their counterparts $\gamma p \rightarrow \gamma^{*} p$ and $M p \rightarrow \gamma^{*} p$ with timelike photons [9]. It turns out that polarization for the photon and meson plays a quite different role than on the target side.

\subsection{The photon side}

To access generalized parton distributions one needs a large virtuality $Q^{2}$ of the virtual photon, and polarization offers a valuable tool to asses whether a given $Q^{2}$ is indeed large enough. This is because in the large limit $Q^{2}$ selection rules emerge for the helicities on the photon side. They follow from two ingredients. First, one can neglect $t$ when evaluating the hard partonic subprocess, where effects of nonzero $t$ are suppressed by a power of $\sqrt{-t} / Q$. The hard scattering thus becomes collinear. The second ingredient is that the chirality of light quarks is conserved in the hard scattering, up to corrections in the tiny current quark masses. The axial anomaly of QCD does not invalidate this argument: chirality violation can occur in quantities dominated by soft physics but not in a hard scattering kernel 10 .

Taking the Compton diagram in Fig. Tha as an example, we thus have that the hard scattering, including radiative corrections, cannot change the quark helicity. In the collinear limit helicity is equivalent to angular momentum along the collision axis, so that the photon helicity must be conserved, too. The leading Compton amplitudes thus have a transverse initial photon, whereas the ones with a longitudinal $\gamma^{*}$ are subleading in $1 / Q$. For meson production one finds that the leading amplitude is the one where both the $\gamma^{*}$ and the meson have helicity zero 11 .

These selection rules can be tested experimentally. Consider the electroproduction processes $e p \rightarrow e p \gamma$ or $e p \rightarrow e p M$ in the targe rest frame. The azimuthal angle $\varphi$ between the planes spanned by the initial and scattered lepton and by the outgoing proton and photon (meson) describes a rotation around the direction of the intermediate $\gamma^{*}$. Quantum mechanics tells us that rotations are intimately connected with angular momentum, and it is not surprising that the $\varphi$ distribution contains information about the $\gamma^{*}$ helicity. If the meson $M$ decays, as in $\rho \rightarrow \pi^{+} \pi^{-}$, further information can be gained from its decay angular distribution.

An application of these results is the $\sin \varphi$ dependence of the single electron spin asymmetry in pion electroproduction measured by HERMES [12]. It directly tells us that amplitudes with a longitudinal and a transverse $\gamma^{*}$ have 
interfered. Since the asymmetry is rather large we can infer that a subleading amplitude (the one with a transverse photon) is not negligible compared with the leading one for the process and $Q^{2}$ range in question. The angular analysis in deeply virtual Compton scattering is more involved [13, but up to corrections a $\sin \varphi$ behavior of the single electron spin asymmetry comes from transverse and a $\sin (2 \varphi)$ from longitudinal $\gamma^{*}$. Both the HERMES and CLAS data [14] shows little sign of a $\sin (2 \varphi)$ variation and thus suggests that the amplitudes with a longitudinal $\gamma^{*}$ are indeed suppressed.

The arguments sketched above can be extended beyond the leading approximation in $1 / Q$. For Compton scattering this has been worked out, and one finds that at the level of $1 / Q$ corrections one only has longitudinal $\gamma^{*}$ polarization. This is twice good news: for one thing it means that $1 / Q$ corrections (which need not be tiny at moderate $Q^{2}$ ) do not affect the $\sin \varphi$ part of the single spin asymmetry from which one wants to extract the leading amplitudes. On the other hand, when data is sufficiently accurate to extract the $\sin (2 \varphi)$ component one will be able to study the first subleading amplitudes for themselves. This would be interesting because they can still be expressed in terms of generalized parton distributions at twist-three level, in a manner very similar to the polarized structure function $g_{2}(x)$ in inclusive deep inelastic scattering [15].

\subsection{The target side}

Unless the transverse momentum transfer $\left(p^{\prime}-p\right)_{\perp}$ is exactly zero, the polarizations of the initial and final proton do not follow selection rules like the photon and meson, they are indeed unconstrained by the helicities on the photon side. This is because a momentum transfer of a few $100 \mathrm{MeV}$ is small compared with $Q$, but not compared with a typical hadronic scale governing the physics of the hadronic matrix elements in our factorized diagrams. The overall helicity of the process need not be conserved since transfer of orbital angular momentum will ensure angular momentum conservation. This is another example of what we saw in the first section: by going away from the strictly collinear kinematics of the usual parton model quantities we get access to physics in the transverse direction, and a particular aspect of this is the orbital angular momentum along the longitudinal axis.

The wave function representation tells us for instance that the generalized parton distribution $E$ (and thus also its first moment, the Pauli form factor $F_{2}(t)$ ) would vanish if the proton did not have configurations where the helicities of the partons do not add up to the helicity of the proton. This reflects from a quite different angle the statement of Ji's sum rule [2] which connects $E$ with the orbital angular momentum of quarks in the proton.

Since the hadron helicities are not fixed, a cross section in general measures a particular combination of initial and final proton helicities, and thus of the associated generalized parton distributions. In the large $Q^{2}$ limit, the beam 
charge asymmetry in Compton scattering involves for instance the combination

$$
\cos \varphi\left[F_{1}(t) H+\xi\left(F_{1}(t)+F_{2}(t)\right) \tilde{H}-\frac{t}{4 M_{p}^{2}} F_{2}(t) E\right]
$$

of Ji's distributions ( $\tilde{E}$ drops out of this observable by an accident of symmetry). Notice that one cannot use the trick of Rosenbluth separation here: to vary the factors weighting the distributions in (1) one must vary $t$ or $\xi$, but the distributions themselves depend on these variables. On the other hand, the full calculation [16] shows that with longitudinal and transverse target polarization (but without measuring recoil polarization) one obtains enough linear combinations of the four distributions to separate them in principle.

\section{Generalized transversity distributions}

Quark transversity distributions, which in a helicity basis describe quark helicity flip, are interesting objects for several reasons 17. Little is known about their size and shape, and vigorous experimental effort will be devoted to improve this situation in the coming years. These distributions have of course a generalization to non-forward kinematics [18. Since helicity conservation does not apply at nonzero $\left(p^{\prime}-p\right)_{\perp}$ there are four distributions for each quark flavor, corresponding to the four helicity combinations of the initial and final proton [19]. Time reversal invariance does not eliminate any of these, but rather fixes their phase and determines their behavior under $\xi \rightarrow-\xi$. The counting of generalized parton distributions for a given parton species and target is thus different from the one for helicity amplitudes or for forward distributions.

Unfortunately no process is presently known where generalized quark transversity occurs, but the situation is better for the generalized helicity flip distributions with gluons. Note that these exist even for spin $\frac{1}{2}$ targets, unlike their forward counterparts, where the gluon helicity flip by two units must be matched on the target side. A remarkable property of these distributions is that under evolution they do not mix with quarks (which cannot flip helicity by two units). Gluon transversity distributions can thus not be generated by DGLAP-type gluon radiation off quarks and in this sense probe "intrinsic glue" in the target. Next to nothing is known about them, but we can infer on their behavior at very small momentum fractions from their evolution equations. For this it is enough to consider the forward case (with for instance a photon target to satisfy helicity conservation) [20]. In the double logarithmic limit of large $\log Q^{2} \log (1 / x)$ one obtains

$$
g(x) \sim x^{-1} f_{1}(x), \quad \Delta g(x) \sim x^{0} f_{2}(x), \quad \delta g(x) \sim x^{1} f_{3}(x),
$$

for the unpolarized, longitudinally polarized, and transversity distributions, respectively, with the residual $x$ dependence in the functions $f_{i}(x)$ controlled by $\alpha_{s}$. Results of the double logarithmic approximation should be used with care, but the hierarchy in (2) does suggest that gluon transversity will fall and not 
rise at small $x$. The region of moderate momentum fractions may thus be the best place to look for these distributions.

Gluon transversity occurs in deeply virtual Compton scattering, where to leading order in $1 / Q$ it provides the only contribution to the photon double helicity flip amplitude. It thus comes with a characteristic signature in the $\varphi$ distribution, which provides a handle to separate it from other distributions. The beam charge asymmetry to leading order in $1 / Q$ for instance goes like

$$
\cos 3 \varphi \frac{\alpha_{s}}{\pi}\left[F_{2}(t) H_{T}-2\left(F_{1}(t)+\frac{t}{4 M_{p}^{2}} F_{2}(t)\right) \tilde{H}_{T}-F_{1}(t) E_{T}\right],
$$

where $H_{T}, \tilde{H}_{T}, E_{T}$ are three of the four gluon transversity distributions. Unfortunately this contribution has to compete with terms suppressed by $M_{p}^{2} / Q^{2}$ but without the factor $\alpha_{s} / \pi$ 21]. Whether gluon transversity can be accessed in Compton scattering will thus depend on their size and on the achievable values of $Q^{2}$. Another process recently proposed is $\gamma^{*} p \rightarrow \rho p$ [22]. Here gluon transversity appears in the amplitudes with double helicity flip from the photon to the $\rho$, which are subleading in the $1 / Q$ expansion.

\section{Deuteron target}

Some of the facilities that can study virtual Compton scattering and meson production run with deuteron targets a part of their time, and it is natural to ask what can be learned from the corresponding generalized parton distributions.

The number of helicity combinations for a spin-one target is rather large, but the algebra involved in the appropriate distributions is not hopelessly tedious [23]. Concentrating on the parton helicity conserving sector, a counting argument analogous to the one we made for the proton tells us that for each parton species there are 9 generalized distributions, 5 of them averaged over parton helicities and 4 sensitive to parton polarization.

In the forward limit three of the quark distributions tend to the well-known quark densities in the deuteron, which at leading order in $\alpha_{s}$ are proportional to the structure functions $F_{1}(x), b_{1}(x)$, and $g_{1}(x)$. Taking the lowest moment in $x$ we recover the 3 vector and the 2 axial vector form factors for elastic deuteron transitions from 5 of the generalized distributions. The lowest moments of the other 4 distributions turn out to be zero for different symmetry reasons, one of them being directly related to the sum rule for $b_{1}(x)$ found by Close and Kumano [24].

The generalized parton distributions of a nuclear system are interesting beyond their spin structure. Consider the simple picture of a deuteron as a weakly bound state of a proton and a neutron, described by two-body wave functions with the appropriate momentum and spin dependence. As for usual parton densities, the parton distribution in the deuteron is then a convolution of these nuclear light-cone wave functions and the parton distributions in a single nu-

cleon, as shown in Fig. 2a and $2 \mathrm{~b}$. In this picture the momentum transfer of 
the process is entirely taken by the emitting nucleon. For nonzero $\xi$ the longitudinal momentum fraction of this nucleon in the bound state before and after the scattering is thus different, and the $\xi$ dependence of the generalized parton distribution reflects rather directly the width of the deuteron wave function in longitudinal momentum. This dependence is again accessible in correlation with the transverse distribution, controlled by the variable $t$. Since the deuteron is a weakly bound system, its wave function will not allow too large values of longitudinal transfer $\xi$. For larger $\xi$ one will thus be sensitive to quantum fluctuations of the deuteron that are more complicated than a system of two almost free nucleons.

(a)

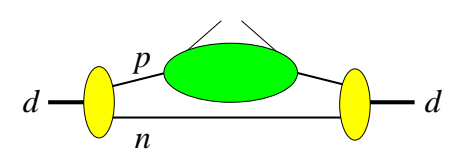

(b)

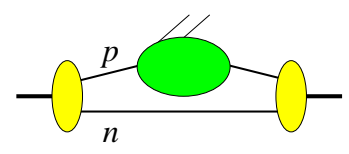

(c)

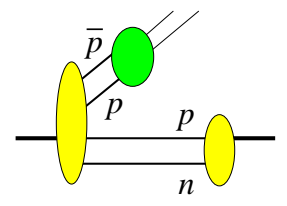

Figure 2: Different contributions to the generalized parton distributions of the deuteron in a convolution model.

The story does not end here. In analogy to the quark-antiquark region of Fig. 1 b, there is now the case where a nucleon-antinucleon pair in the initial deuteron wave function annihilates into a $q \bar{q}$ system which is then emitted (Fig. 2c). The annihilation is described by a generalized distribution amplitude, which can be studied in other hard processes [1, 25. Intuition suggests that four-nucleon configurations should be extremely rare in the deuteron, but they are required by Lorentz invariance. Whereas in the description of the elastic form factors one can avoid their appearance by choosing a frame with $\xi=0$, we now have nonzero $\xi$ imposed by the process. In this sense generalized parton distributions may provide a handle to access truly relativistic effects in a nuclear system.

\section{Conclusions}

Spin plays a variety of roles in the context of generalized parton distributions. In the hard processes processes where these quantities appear, the helicity of the photon and meson provides a handle on the reaction mechanism, which selects certain helicity transitions. Measurement of the corresponding azimuthal distributions allows one to separate different hard scattering contributions. On the other hand, the helicities in the hard subprocess do not fix those of the initial and final hadron, and to separate their different combinations in the parton distributions one needs target (or recoil) polarization.

A particular selection of photon helicities gives access to gluon transversity distributions, provided power correction terms are sufficiently small in the region 
under study. Since they cannot be generated from quarks via perturbative quark-gluon splitting, these distributions constitute a rather unique probe of "intrinsic glue" in the proton. For spin $\frac{1}{2}$ targets they decouple in the forward limit and are only visible in exclusive processes, when helicity is not conserved.

The non-conservation of helicity in generalized parton distributions is what makes these quantities sensitive to orbital angular momentum in an essential way. This is possible because one goes away from the purely collinear kinematics describing the familiar twist-two quantities of the parton model, and illustrates a characteristic feature of nonforward exclusive processes: they simultaneously probe the transverse and the longitudinal distribution of quarks and gluons in a hadron state. For nuclear systems we have seen that, in analogy, one may be able to access the longitudinal and transverse distribution of its nucleon constituents, and possibly more complicated configurations.

Clearly, the dependence of generalized parton distributions on three kinematical variables, and the number of distributions describing different helicity combinations present a considerable complexity. In a sense this is the price to pay for the amount of physics information encoded in these quantities. It is however crucial to realize that for many important aspects one need not fully disentangle this complexity. The interrelation of longitudinal and transverse structure of partons in a nucleon, or of nucleons in a nucleus, can be studied quantitatively from the distribution in the two external kinematical variables $\xi$ and $t$, even if the deconvolution of the loop integral over the variable $x$ is not performed. In a similar way, the very information of whether gluon transversity is small or large for a typical size of longitudinal momentum set by $\xi$ would in itself be valuable, given how little we know about the non-perturbative origin of quark and gluon distributions in QCD bound states.

\section{Acknowledgements}

It is a pleasure to thank Bo-Qiang Ma and his colleagues for the marvelous organization of this meeting, and Bernard Pire for valuable remarks on the manuscript.

\section{References}

[1] D. Müller et al., Fortsch. Phys. 42, 101 (1994) hep-ph/9812448.

[2] X. Ji, Phys. Rev. Lett. 78, 610 (1997) hep-ph/9603249.

[3] A. V. Radyushkin, Phys. Rev. D56, 5524 (1997) hep-ph/9704207.

[4] J. Blümlein et al., Nucl. Phys. B 560, 283 (1999) hep-ph/9903520.

[5] M. Burkardt, Phys. Rev. D 62, 071503 (2000) hep-ph/0005108.

[6] J. P. Ralston and B. Pire, hep-ph/0110075. 
[7] S. J. Brodsky et al., Nucl. Phys. B 596, 99 (2001) hep-ph/0009254;

M. Diehl et al., Nucl. Phys. B 596, 33 (2001) hep-ph/0009255.

[8] S. J. Brodsky, P. Hoyer, N. Marchal, S. Peigné and F. Sannino, hep$\mathrm{ph} / 0104291$

S. J. Brodsky, these proceedings.

[9] E. R. Berger, M. Diehl and B. Pire, hep-ph/0110062;

Phys. Lett. B 523, 265 (2001) hep-ph/0110080.

[10] J. C. Collins and M. Diehl, Phys. Rev. D 61, 114015 (2000) hep$\mathrm{ph} / 9907498$;

P. Hoodbhoy, hep-ph/0108214.

[11] J. C. Collins et al., Phys. Rev. D 56, 2982 (1997) hep-ph/9611433;

M. Diehl, T. Gousset and B. Pire, Phys. Rev. D 59, 034023 (1999) hep$\mathrm{ph} / 9808479$.

[12] A. Airapetian et al. [HERMES Collaboration], hep-ex/0112022.

[13] M. Diehl et al., Phys. Lett. B411, 193 (1997) hep-ph/9706344.

[14] A. Airapetian et al. [HERMES Collaboration], Phys. Rev. Lett. 87, 182001 (2001) hep-ex/0106068];

S. Stepanyan et al. [CLAS Collaboration], Phys. Rev. Lett. 87, 182002 (2001) hep-ex/0107043.

[15] I. V. Anikin et al., Phys. Rev. D 62, 071501 (2000) hep-ph/0003203;

A. V. Belitsky and D. Müller, Nucl. Phys. B 589, 611 (2000) hep$\mathrm{ph} / 0007031$

A. V. Radyushkin and C. Weiss, Phys. Rev. D 63, 114012 (2001) hep$\mathrm{ph} / 0010296$.

[16] A. V. Belitsky, D. Müller and A. Kirchner, hep-ph/0112108.

[17] R. L. Jaffe, hep-ph/0102281.

[18] P. Hoodbhoy and X. Ji, Phys. Rev. D58, 054006 (1998) hep-ph/9801369];

A. V. Belitsky and D. Müller, Phys. Lett. B486, 369 (2000) hep$\mathrm{ph} / 0005028$.

[19] M. Diehl, Eur. Phys. J. C 19, 485 (2001) hep-ph/0101335.

[20] B. Ermolaev et al., Eur. Phys. J. C 7, 65 (1999) hep-ph/9806439.

[21] N. Kivel and L. Mankiewicz, hep-ph/0106329.

[22] N. Kivel, hep-ph/0107275.

[23] E. R. Berger et al., Phys. Rev. Lett. 87, 142302 (2001) hep-ph/0106192.

[24] F. E. Close and S. Kumano, Phys. Rev. D 42, 2377 (1990).

[25] M. Diehl et al., Phys. Rev. Lett. 81, 1782 (1998) [hep-ph/9805380]. 